\title{
Internet-Based Virtual Computing Environment
}

\author{
Huaimin Wang \\ National Lab. For Parallel and Distributed Processing \\ National University of Defense Technology \\ Changsha, Hunan, P.R. China
}

Different from the traditional resources, resources over the Internet have such natural characteristics as growth, autonomy and diversity, which have brought grand challenges to their efficient sharing and comprehensive utilization. As there are the essential differences between the Internet computing environment and the traditional computer environment, the resource management methods for traditional computers is no longer suitable for the internet any more. The emergence and development of Web 2.0 and P2P technologies in recent years manifests that the resource management over Internet should adapt to the natural characteristics of the Internet resources and virtualization is one of the potential means to deal with those challenges. However, virtualization in Internet computing environment is essentially different from that in traditional computer environment. There are three scientific issues to be explored, i.e., on-demand aggregation in the open Internet environment, autonomic collaboration among distributed autonomous resources, computational properties of the aggregation and collaboration. We propose the Internet-based Virtual Computing Environment (iVCE), which aims at providing harmonious, trustworthy and transparent computing environment and services for end-users and applications by the means of novel computing models and innovative mechanisms, such as the on-demand resource aggregation and autonomic resource collaboration. The iVCE tries to virtualize and autonomicize Internet resources, design new models and mechanisms adapting well to the Internet computing environment, and promote the sharing and utilization of the Internet resources. Many progresses have been achieved in the model, technical framework and mechanisms of iVCE in the past several years. The concept model of iVCE includes autonomic element (AE), virtual commonwealth (VC) and virtual executor (VE). The architecture of iVCE is designed based on these models. Many mechanisms have been proposed in such areas as resource virtualization, on-demand aggregation, autonomic collaboration, trustworthy enhancement and programming language facility. The iVCE middleware has been designed and implemented, providing a set of services to support the flexible sharing and collaboration of Internet resources. The iVCE middleware has also provided some tools, such as virtual network storage, virtual network memory and virtual cluster for users. Many applications have been run on the iVCE middleware. iVCE will support the multi-scale sharing and collaboration of resources in various granularities, including the large-scale data center, client resources and various resources on the edge of Internet. The productivity and trustworthiness of iVCE will be focused on to investigate in the future. The integration of multiple virtual commonwealths is also part of our future work. 